\title{
Vapor Activity and Systemic Movement of Mefenoxam Control Grapevine Downy Mildew
}

\author{
Megan M. Kennelly, David M. Gadoury, Wayne F. Wilcox, and Robert C. Seem, Department of Plant Pathology, \\ Cornell University, New York State Agricultural Experiment Station, Geneva 14456
}

\begin{abstract}
Kennelly, M. M., Gadoury, D. M., Wilcox, W. F., and Seem, R. C. 2007. Vapor activity and systemic movement of mefenoxam control grapevine downy mildew. Plant Dis. 91:1260-1264.

Metalaxyl is translocated from roots to leaves to control a number of oomycete pathogens, but systemic movement from vegetative organs into fruit and vapor activity against Plasmopara viticola, the causal agent of grapevine downy mildew, has not been examined experimentally. We inoculated fruit clusters of grapevines with $P$. viticola at prebloom, bloom, or 1 week postbloom. We then selectively applied mefenoxam ( $288 \mathrm{mg} / \mathrm{liter})$, the active enantiomer of metalaxyl, to the leaves or stem tissue 12 to $48 \mathrm{~h}$ after inoculation. Little to no downy mildew developed on fruit when mefenoxam was applied to leaf tissue, stem tissue, or both. In contrast, downy mildew symptoms were severe on inoculated clusters on untreated shoots. When potential vapor activity was blocked, we observed fungicidal activity on seedling foliage in response to apparent systemic movement from treated stems and soil, but not from leaves. However, when vapor activity was permitted, mefenoxam residues on treated leaves controlled disease on other, untreated leaves. In subsequent vineyard experiments, vapor and systemic activity provided equivalent and near-complete suppression of downy mildew on clusters $48 \mathrm{~h}$ post inoculation. Furthermore, inoculated grape seedlings that were placed near mefenoxam-treated seedlings in open and closed systems developed nil to trace levels of downy mildew compared with controls, further indicating that the material has strong vapor activity.
\end{abstract}

Additional keyword: Ridomil

Physical mode of action refers to the effect of the timing or placement of a fungicide on efficacy. Szkolnik (10) used this term to describe temporal aspects of fungicide action (pre-infection, post-infection, pre-symptom, or post symptom), as well as spatial factors such as vapor activity. The physical mode of action of a fungicide has important implications for the timing and placement of fungicides in a control program. Grapevine downy mildew, caused by Plasmopara viticola (Berk. \& M. A. Curtis) Berl. \& De Toni, is controlled primarily by fungicides (7), including mefenoxam (the active " $R$ " enantiomer of metalaxyl). Knowledge of the physical modes of action of fungicides can improve our ability to use them.

Metalaxyl has been shown to move systemically in many plants via acropetal and localized translocation by bulk-flow with water in xylem tissue $(1,8)$. For the purposes of this study, it is assumed that me-

Corresponding author: M. M. Kennelly E-mail: kennelly@ksu.edu

Current address of M. M. Kennelly: Department of Plant Pathology, Kansas State University, Manhat$\tan 66506$.

Accepted for publication 27 April 2007.

doi:10.1094/PDIS-91-10-1260

(c) 2007 The American Phytopathological Society fenoxam shares similar properties with metalaxyl. Staub et al. (9) studied systemic movement of metalaxyl in grapevine seedlings for control of downy mildew, and demonstrated that there was effective translocation to the upper leaves following a soil drench or direct application to the main stem. However, this fungicide did not move from treated lower leaves to untreated upper leaves in quantities sufficient to control disease (9). Similarly, metalaxyl applied to tomato leaves did not control Phytophthora infestans on any other leaves, either above or below the treated one, or in the fruit (2). In addition, cultures of $P$. parasitica and $P$. splendens were inhibited by metalaxyl vapor in closed containers $(3,6)$.

Wong and Wilcox (11) examined the physical mode of action of metalaxyl against downy mildew on grapevine seedlings. The fungicide significantly reduced colony area and sporulation when applied up to 5 days after infection, and also provided complete disease control on the abaxial leaf surface following preinoculation treatment of only the adaxial surface. Although this latter phenomenon was interpreted as evidence for translaminar movement, the possibility of vapor activity was not excluded.

There have been no controlled studies to examine translocation of mefenoxam into fruit clusters of mature grapevines or the vapor activity of this fungicide against
Plasmopara viticola. In several field experiments in which we protected clusters with plastic bags during mefenoxam applications, disease often failed to develop on the putatively fungicide-free clusters after they were inoculated, suggesting that the material might have translocated into fruit clusters from shoot or leaf tissue, or that it exhibited significant vapor activity. Thus, our initial objective in the present study was to determine whether mefenoxam can protect unsprayed grape clusters through systemic or vapor movement from treated shoot tissue. Subsequently, we also sought to determine whether this movement was possible from leaf or stem tissue, and whether it effectively controlled downy mildew in the fruit when applications were made up to $48 \mathrm{~h}$ after inoculation.

\section{MATERIALS AND METHODS}

Vineyard studies were performed on $\mathrm{Vi}$ tis vinifera L. cvs. Chardonnay and Riesling and Vitis interspecific hybrid cv. Seyval at the New York State Agricultural Experiment Station (NYSAES) in Geneva, NY, and on V. labrusca L. cv. Niagara at a commercial vineyard in Dresden, NY. The Chardonnay/Riesling/Seyval vineyard was planted in 1974 with $2.4 \mathrm{~m}$ between vines and $2.7 \mathrm{~m}$ between rows, and vines were Umbrella-Kniffen trained and cane pruned. The Niagara vineyard was planted with 2.9 $\mathrm{m}$ between rows and $2.3 \mathrm{~m}$ between vines and was cordon trained and mechanically hedge pruned. Seedling experiments were performed in the laboratory and greenhouse on open-pollinated Riesling seedlings in $6 \mathrm{~cm}$-diameter pots containing a potting mix of Cornell Mix and perlite (3:1, vol/vol).

Inoculum production and inoculation. Inoculation procedures were based on previously described methods $(4,5)$. Inoculum consisted of sporangial suspensions prepared from grapevine seedlings (five- to six-leaf stage) that had been inoculated 1 week earlier. The original source was a mixture of field isolates from natural infections in the experimental vineyard. Inoculated seedlings were incubated at $100 \%$ relative humidity (RH) overnight at room temperature and, the next day, sporangia were rinsed from the lower surface of the leaves using a stream of distilled water, which was collected in a beaker and then adjusted to a concentration of $8 \times 10^{4}$ to 1 $\times 10^{5}$ sporangia $/ \mathrm{ml}$. We sprayed the sporangial suspension onto inoculated tissues 
using a hand-held spray bottle, and enclosed them overnight in wet plastic bags sealed with a plastic-coated wire. In the field, inoculations were performed at dusk or on cloudy afternoons to ensure the bags did not overheat in direct sunlight, and the wet bags were removed the following morning within $3 \mathrm{~h}$ of sunrise. For seedling experiments, entire plants were enclosed in plastic bags after inoculation for approximately $12 \mathrm{~h}$.

Spore suspensions were used within $1 \mathrm{~h}$ of preparation. We checked inoculum quality by placing a drop of the spore suspension onto a microscope slide and assessing the percentage of sporangia that had produced zoospores 2 to $12 \mathrm{~h}$ after preparing the suspension. In all cases, at least $75 \%$ of sporangia produced zoospores.

In all experiments, a commercial formulation of mefenoxam (Ridomil Gold EC; Syngenta Crop Protection Inc, Greensboro, NC) was applied at the rate of $288 \mathrm{mg}$ a.i./liter, equivalent to the rate of mefenoxam registered for use to control grapevine downy mildew in the United States.

Vineyard experiments. Systemic activity evaluations on mature vines, 2002 and 2003. The fungicide was applied to the leaves and the stem, only the leaves, or only the stem, on four to five nodes centered on an inoculated cluster. For convenience, these treatments were referred to as leaf + stem, leaf, and stem. For leaf + stem applications, we used a hand-held spray bottle to apply the material to run-off. For applications to just the leaves or stems, we used a small paintbrush to coat the appropriate tissues until run-off. During fungicide applications, inoculated flower or fruit clusters were enclosed in plastic bags sealed with a plastic-coated wire to shield them from direct contact with the material.

The experimental unit was one vine, with all treated shoots on each vine receiving a leaf + stem, leaf, or stem application. Chardonnay and Riesling treatments were assigned randomly in dispersed vines within the mixed planting described above. Due to space and practical constraints in the commercial vineyard, Niagara fungicide treatments were assigned within 6 to 8 contiguous vines, with the untreated controls randomly assigned within 55 contiguous vines further down in the same row. On each shoot, we inoculated the basal cluster. For controls, we inoculated clusters on vines not treated with fungicides. Unless otherwise stated, in all experiments there were four shoots on each of three or four replicate vines per treatment, and four shoots on each of four replicate vines for the control treatment.

In 2002, Chardonnay and Niagara clusters were inoculated 16 and 14 days prebloom, respectively, and leaf + stem treatments were made 12 to $20 \mathrm{~h}$ later. Clusters on additional shoots were inoculated at bloom (50\% flowers open) and leaf + stem treatments were made 42 to $48 \mathrm{~h}$ later.
In 2003, Niagara clusters were inoculated either 8 days prebloom or at bloom, and leaf + stem applications were made 18 h later. In 2003, we also carried out leaf + stem, leaf, and stem treatments on Chardonnay and Riesling to determine from which tissues the fungicide was moving. Clusters of both cultivars were inoculated 16 days prebloom and mefenoxam was applied $22 \mathrm{~h}$ later. Additional Chardonnay clusters were inoculated 1 week postbloom, and mefenoxam was applied $12 \mathrm{~h}$ later. We examined two shoots per vine on each of three replicate vines per treatment.

Disease severity was assessed 2 weeks after inoculation. Because both darkness and high $\mathrm{RH}$ are required for sporulation of $P$. viticola, we misted the insides of clear plastic bags with water, fastened the bags around the clusters, and tied the bags closed overnight with a plastic-coated wire. The following morning, we removed the plastic bags and estimated the percentage of cluster surface supporting sporulation. Though downy mildew causes discoloration and necrosis on older clusters (4), sporulation is abundant on clusters inoculated at the growth stages used.

Differentiation of systemic from vapor activity. To assess systemic activity in the absence of the vapor phase, Seyval clusters were inoculated on 21 June 2005 (bloom), as described above. Inoculated clusters were covered $48 \mathrm{~h}$ later with a plastic bag that was loosely secured around at the base of the rachis with a plastic-coated wire, and fresh air was supplied through a 0.6$\mathrm{cm}$-diameter plastic tube connected to a battery-operated blower, which provided a complete change of air within the bag every $5 \mathrm{~s}$. Replacement air for the blower was drawn through a 16-mm-diameter hose approximately $22 \mathrm{~m}$ from the treated tissues. Each of these clusters was shielded from direct sunlight by covering it with a loosely fitting white paper bag to prevent heat build-up in the plastic bag. Mefenoxam was applied to $4 \mathrm{~cm}$ of stem tissue on each of two internodes immediately below (treatment 1) or above (treatment 2) the inoculated clusters. Inoculated control clusters were bagged similarly, but were located on vines not treated with mefenoxam. All treatments were replicated five times, with the second cluster of a single shoot, each located on separate vines, serving as the experimental unit. All inoculated clusters were removed from the vines on 28 June and incubated at $25^{\circ} \mathrm{C}$ and $100 \% \mathrm{RH}$ overnight, and the percentage of the cluster surface supporting sporulation of $P$. viticola was estimated visually. The experiment was repeated as above on clusters inoculated on 29 June (7 days postbloom).

Vapor activity alone was assessed by selecting paired shoots on adjacent Chardonnay vines. On 21 June (midbloom), the basal cluster of one shoot was inoculated as described above. On 23 June (48 h post inoculation), the inoculated shoot was covered with a loosely fitting plastic bag while mefenoxam was sprayed to run-off on all leaves of the paired shoot, using a hand-held atomizer (Preval C132 Sprayer; Precision Valve Corp., Yonkers, NY). Once the spray deposit had dried, the plastic bag was removed and each shoot was tied individually to one of two vertical parallel wires spaced approximately $13 \mathrm{~cm}$ apart. Control clusters on separate vines were inoculated and similarly trained parallel to unsprayed shoots of an adjacent vine. Treatments were replicated five times as paired shoots (inoculated and sprayed or inoculated and unsprayed) borne on pairs of adjacent vines. All inoculated clusters were removed from the vines on 28 June and incubated at $25^{\circ} \mathrm{C}$ and $100 \%$ RH overnight, and the percentage of the cluster surface supporting sporulation of $P$. viticola was estimated visually. The experiment was repeated as above on clusters inoculated on 29 June (7 days postbloom). A datalogger (CR10; Campbell Scientific Inc., Logan, Utah) at the vineyards provided records of rainfall, temperature, surface wetness duration, and RH.

Seedling experiments. Evaluation of vapor and systemic activity. We inoculated the three youngest fully expanded leaves on seedlings and applied mefenoxam $24 \mathrm{~h}$ later to the middle leaf, basal stem tissue beneath the first node, or potting mix. The plants then were placed singly in clear, plastic 2-liter soft drink bottles modified to expose specific tissues to the fungicide. For some treatments, the interior of the bottle was infiltrated with two to four exchanges per minute of filtered air (Sharpe 880A 1.0- $\mu \mathrm{m}$ particulate filter; Sharpe Spray Systems, Santa Fe Springs, CA) to create a positive pressure and ensure that no mefenoxam vapor from extraneous sources could reach the leaves inside, while other parts of the plant remained outside the bottle. In the first experiment, the specific treatments were (i) no mefenoxam, entire seedling inside bottle, no airflow; (ii) mefenoxam-treated leaf outside bottle, with airflow; and (iii) mefenoxam-treated leaf inside bottle, no airflow. We repeated the experiment two additional times, adding the following treatments: (iv) mefenoxam-treated leaf inside bottle, with airflow; (v) mefenoxamtreated stem outside bottle, with airflow; and (vi) mefenoxam-treated potting mix (35 $\mu \mathrm{g}$ a.i. $/ \mathrm{cm}^{3}$ of potting mix, applied in a nonsaturating drench outside the bottle perimeter), with airflow. There were four seedlings per treatment, each in a separate bottle. Treatments 1, 2, and 3 were performed three times. Treatments 4, 5, and 6 were performed twice. Except for the soil drench, mefenoxam was applied with a small paintbrush and care was taken to prevent fungicide dripping onto nontarget tissues or soil. 
In another seedling experiment, we inoculated the five youngest fully expanded leaves, then applied mefenoxam to the basal or distal two leaves $24 \mathrm{~h}$ later, or left them untreated. We placed the seedlings in plastic trays, with one inoculated, untreated seedling; one seedling with treated basal leaves; and one seedling with treated distal leaves in each tray, spaced so that they were not touching. We placed three trays individually inside clear plastic bags, which subsequently were sealed with a plastic-coated wire, and left three trays open. Additionally, we had six trays of untreated, inoculated seedlings (three enclosed in bags, three open) as controls. Open trays with treated seedlings were placed at least $2 \mathrm{~m}$ apart, and the control trays were placed in another room to prevent any exposure to mefenoxam vapor. This experiment was performed twice. The experiment was repeated a third time with the following modifications. We inoculated the three youngest fully expanded true leaves and two cotyledons on 12 seedlings. Mefenoxam was applied to the true leaves of eight additional seedlings. One inoculated and one fungicide-treated plant were placed into eight trays (four open, four closed). The remaining four inoculated, untreated plants were placed in trays in a separate room to serve as controls. Disease severity was assessed 1 week after inoculation by placing seedlings in moist chambers overnight and estimating the percent leaf area supporting sporulation the following morning.

\section{RESULTS}

Vineyard studies. In the vineyard studies of 2002 and 2003, mefenoxam provided nearly complete control (98 to $100 \%$ ) of downy mildew on clusters when applied only to leaf or stem tissue 12 to 48 $\mathrm{h}$ after inoculation of the clusters, despite their high susceptibility to infection and regardless of cultivar, the specific tissues treated, or the developmental stage of the crop (Table 1). However, it was not possi- ble to separate vapor activity from systemic activity in these experiments.

In vineyard experiments in 2005 where vapor activity was excluded, mefenoxam moved systemically from stem tissue beneath clusters to provide nearly complete control of downy mildew, but no control was provided when the fungicide was applied to stem tissue above clusters (Fig. 1). In the 2005 vineyard experiment examining vapor activity, treatment of leaf tissue on one shoot provided nearly complete control of downy mildew on clusters of an untreated shoot on an adjacent vine (Fig. 2). Daily high temperatures during the experiment ranged from 20 to $32^{\circ} \mathrm{C}$ while daytime low temperatures ranged from 9 to $20^{\circ} \mathrm{C}$. The average daily temperature was $19.8^{\circ} \mathrm{C}$ during the first run of the experiment and $21.5^{\circ} \mathrm{C}$ during the second run of the experiment. No rain occurred between the time of application of mefanoxam and removal and incubation of the inoculated clusters in either run of the experiment.

Seedling assays. Mefenoxam showed strong systemic movement and complete control of the disease in seedlings when it was applied to the stem or soil and any vapor contact with the plants was excluded (Table 2). However, the chemical displayed strong vapor activity as well. When the fungicide-treated leaf was excluded from the chamber, the inoculated leaves inside, both above and below it, developed severe downy mildew (Table 2). In contrast, the other leaves did not develop any disease when the fungicide-treated leaf was inside the chamber, even when constant air exchange prevented the accumulation of mefenoxam vapor (Table 2). Fur-

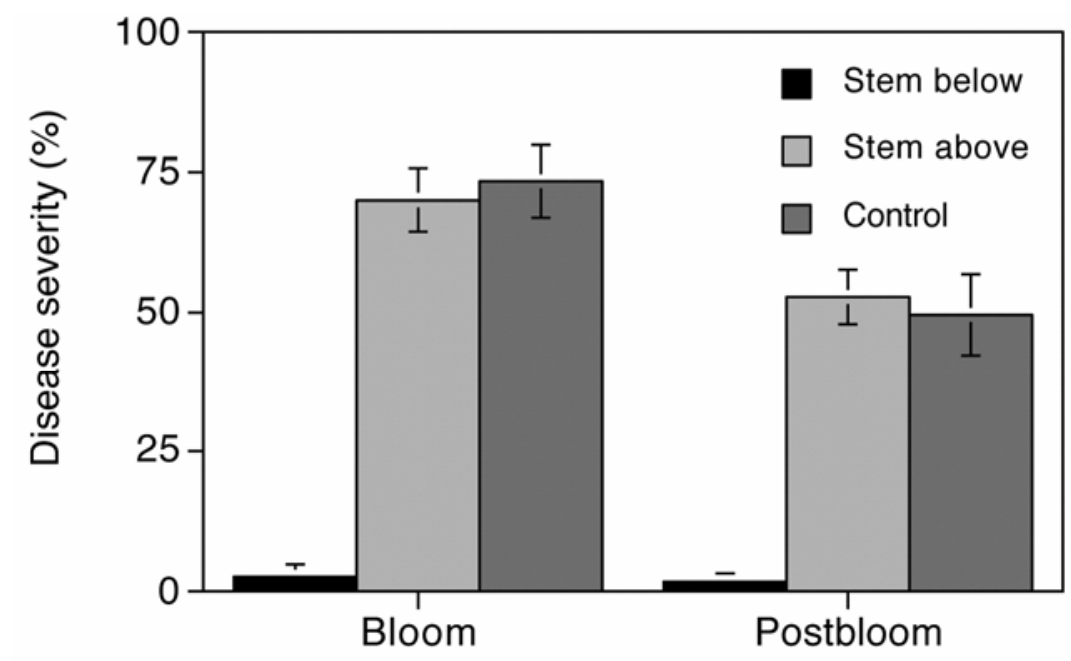

Time of inoculation

Fig. 1. Mefenoxam (288 mg/liter) was applied to $4 \mathrm{~cm}$ of stem tissue on each of two internodes below (Stem below) or above (Stem above) selected clusters $48 \mathrm{~h}$ after inoculation with Plasmopara viticola using a 4-cm-wide paintbrush. Control clusters were inoculated, but were located on vines not treated with mefenoxam. All treatments were replicated five times as single clusters, each located on separate vines. All inoculated clusters were removed from the vines 1 week after mefenoxam application and incubated at $25^{\circ} \mathrm{C}$ and $100 \%$ relative humidity overnight, and the disease severity was determined by estimating visually the percentage of the cluster surface supporting sporulation of $P$. viticola.

Table 1. Effect of mefenoxam application on leaf and stem tissue on downy mildew development on inoculated clusters

\begin{tabular}{|c|c|c|c|c|c|c|}
\hline \multirow[b]{2}{*}{ Year, cultivar } & \multirow[b]{2}{*}{ Timing (hpi) ${ }^{\mathbf{b}}$} & \multirow[b]{2}{*}{ Vine stage $^{c}$} & \multicolumn{4}{|c|}{ Disease severity on clusters ${ }^{a}$} \\
\hline & & & Leaf & Stem & Leaf + stem & Untreated control \\
\hline \multicolumn{7}{|l|}{2002} \\
\hline \multirow{2}{*}{ Niagara } & 12 & 14 days prebloom & n.d. & n.d. & $0.0 \pm 0.0$ & $71.4 \pm 16.2$ \\
\hline & 48 & Bloom & n.d. & n.d. & $1.0 \pm 0.6$ & $67.5 \pm 10.3$ \\
\hline \multirow[t]{2}{*}{ Chardonnay } & 12 & 16 days postbloom & n.d. & n.d. & $0.0 \pm 0.0$ & $73.1 \pm 7.1$ \\
\hline & 42 & Bloom & n.d. & n.d. & $0.0 \pm 0.0$ & $18.8 \pm 2.3$ \\
\hline \multicolumn{7}{|l|}{2003} \\
\hline \multirow{2}{*}{ Niagara } & 18 & 8 days prebloom & n.d. & n.d. & $0.0 \pm 0.0$ & $73.8 \pm 10.4$ \\
\hline & 18 & Bloom & n.d. & n.d. & $0.0 \pm 0.0$ & $81.9 \pm 3.1$ \\
\hline \multirow[t]{2}{*}{ Chardonnay } & 22 & 16 days prebloom & $0.0 \pm 0.0$ & $0.0 \pm 0.0$ & $2.5 \pm 4.3$ & $57.5 \pm 6.6$ \\
\hline & 12 & 7 days postbloom & $0.0 \pm 0.0$ & $0.0 \pm 0.0$ & $0.0 \pm 0.0$ & $19.1 \pm 6.4$ \\
\hline Riesling & 22 & 16 days prebloom & $0.0 \pm 0.0$ & $0.0 \pm 0.0$ & $0.0 \pm 0.0$ & $46.3 \pm 16.4$ \\
\hline
\end{tabular}

a Clusters were inoculated and mefenoxam was applied to the indicated tissues on the same shoot. Disease severity was assessed 2 weeks after inoculation by estimating the percentage of the cluster surface supporting sporulation of Plasmopara viticola. Values represent the average of three or four vines per treatment \pm one standard error of the mean. Leaf- and stem-only applications of mefenoxam were not performed in all cases; n.d. $=$ data not determined for this treatment.

${ }^{\mathrm{b}}$ Mefenoxam application timing, hours post inoculation (hpi).

c Vine stage at time of inoculation. 
thermore, untreated seedlings developed zero to trace levels of sporulation on any leaves when they were placed near a fungicide-treated seedling following inoculation, regardless of which leaves the fungicide had been applied to, in both open and enclosed containers (Table 3). Results were similar in the third repetition of the experiment, in which no disease developed seedlings paired with fungicide-treated pared with $17.6 \%$ (standard error $=2.9$ ) leaf surface supporting sporulation on controls not paired with fungicide-treated seedlings.

\section{DISCUSSION} grapevine downy mildew on unsprayed on cotyledons or true leaves on inoculated seedlings in open or closed trays, com-

We found that mefenoxam controls

tissues through both vapor activity and systemic movement from certain treated tissues, and that these two physical modes of action were equally efficacious in laboratory and vineyard trials. In our 2002 and 2003 vineyard experiments, we observed nearly absolute control from the combined impact of systemic and vapor activity when mefenoxam was applied to both stem and leaf tissue, despite near-constant air movement through the vine canopy. Subsequent vineyard experiments designed to segregate these two activities also yielded near-complete control from each one alone, and our laboratory tests showed that vapor activity remained highly effective in the presence of constant, albeit limited, air exchange. Although metalaxyl has been reported to exhibit vapor activity in vitro against Phytophthora parasitica and $P$.

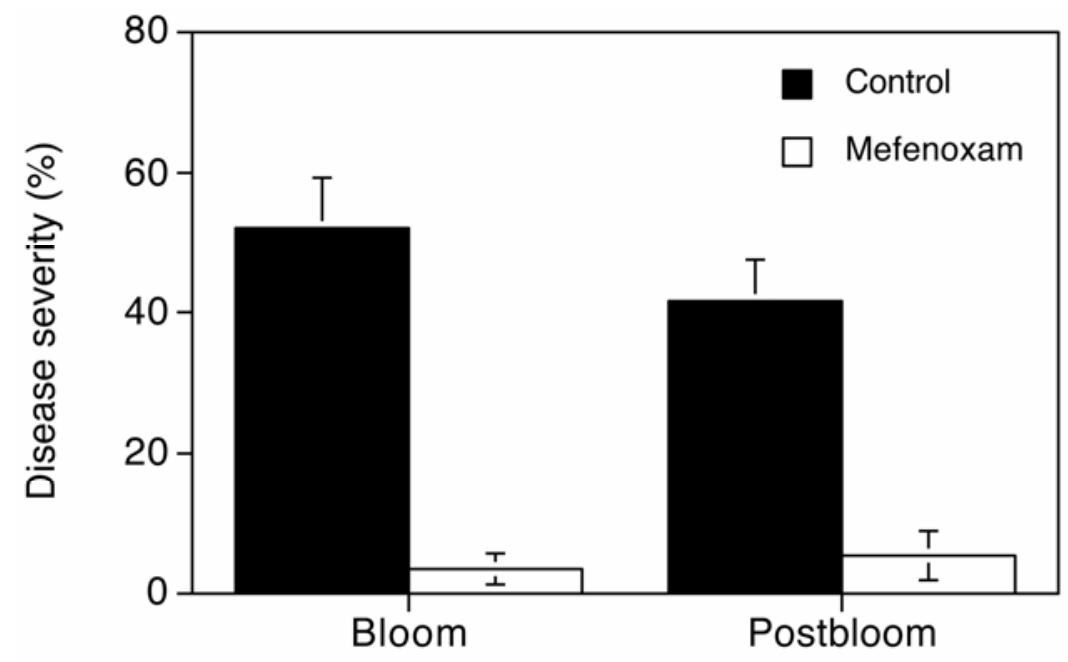

Treatment

Fig. 2. Vapor activity of mefenoxam was assessed by selecting two shoots on adjacent vines of Vitis vinifera cv. Chardonnay. The basal cluster of one shoot was inoculated at the indicated stages of cluster development. At $48 \mathrm{~h}$ postinoculation, the shoot was covered with a loosely fitting plastic bag while mefenoxam was applied to all leaves of a shoot on the adjacent vine. Once the spray deposit had dried, the plastic bag was removed and each shoot was tied to one of two vertical parallel wires, approximately $13 \mathrm{~cm}$ apart. Control clusters on separate vines were inoculated and likewise trained parallel to unsprayed shoots of an adjacent vine. Treatments were replicated five times. All inoculated clusters were removed from the vines 7 days after mefenoxam application and incubated at $25^{\circ} \mathrm{C}$ and $100 \%$ relative humidity overnight, and the disease severity was determined by visually estimating the percentage of the cluster surface supporting sporulation of Plasmopara viticola.

Table 2. Control of downy mildew by mefenoxam on inoculated seedling leaves exposed to various potential sources of vapor or systemic activity

\begin{tabular}{lcrcc}
\hline & & \multicolumn{3}{c}{${\text { Disease severity }(\%)^{\mathbf{a}}}$} \\
\cline { 3 - 5 } Mefenoxam treatment $^{\mathbf{b}}$ & Air flow $^{\mathbf{c}}$ & Top leaf & Middle leaf & Bottom leaf \\
\hline 1. None & No & $63.3 \pm 5.4$ & $67.5 \pm 8.8$ & $65.0 \pm 9.3$ \\
2. Treated leaf outside bottle & Yes & $77.5 \pm 5.3$ & $0.0 \pm 0.0$ & $68.8 \pm 9.6$ \\
3. Treated leaf inside bottle & No & $0.0 \pm 0.0$ & $0.0 \pm 0.0$ & $0.0 \pm 0.0$ \\
4. Treated leaf inside bottle & Yes & $0.0 \pm 0.0$ & $0.0 \pm 0.0$ & $0.0 \pm 0.0$ \\
5. Treated stem outside bottle & Yes & $0.0 \pm 0.0$ & $0.0 \pm 0.0$ & $0.0 \pm 0.0$ \\
6. Soil drench & Yes & $0.0 \pm 0.0$ & $0.0 \pm 0.0$ & $0.0 \pm 0.0$ \\
\hline
\end{tabular}

${ }^{\text {a }}$ Disease was assessed 1 week later by estimating the percentage of leaf tissue with sporulation. Values represent the average of two (treatments 4,5 , and 6 ) or three (treatments 1,2 , and 3 ) repetitions of the experiment, with four seedlings per treatment in each repetition, \pm one standard error of the mean.

${ }^{\mathrm{b}}$ Seedlings were inoculated with Plasmopara viticola and treated with mefenoxam (288 $\mathrm{mg}$ a.i./liter) on the indicated tissues.

${ }^{c}$ Interior of the bottle was infiltrated with two to four exchanges per minute of filtered air. splendens $(3,6)$, the degree to which cluster infections were suppressed under vineyard conditions solely by vapor activity initiated $48 \mathrm{~h}$ post infection was unexpected and appears to represent an underappreciated attribute of the fungicide. This is the first report of vapor activity of mefenoxam or metalaxyl in control of Plasmopara viticola on grape. Based on these findings, it does not seem possible to exclude it as an alternative explanation for the translaminar activity of metalaxyl reported by Wong and Wilcox (11) on grapevine seedlings contained within in a closed system.

Our results are consistent with prior reports in grape (9) and other plants $(2,5,8)$ that the chemical translocates from the stem and via root uptake from soil drenches to control disease in distal parts of the plant. Mefenoxam is thought to follow the transpiration stream in the xylem and, thus, would not be expected to flow basipetally. Zaki et al. (12) used radioactively labeled metalaxyl to study translocation in Persea indica and tomato. When metalaxyl was applied to a single leaf, trace levels (0 to $5 \%$ ) of $\mathrm{C}^{14}$ were detected in leaves above and below and even in the roots, but it was unclear whether this radioactivity was associated with metalaxyl or with some subsequent metabolite. We found no evidence that mefenoxam can move basipetally in grapevines. Rather, mefenoxam moved acropetally from roots to all seedling leaves, and from treated stem tissue to distal leaves. However, it did not move from leaf to leaf, which would require some basipetal movement down the petiole.

Knowledge of the physical mode of action of fungicides can help in the develop-

Table 3. Control of downy mildew on grapevine seedlings by mefenoxam vapor activity

\begin{tabular}{lcc}
\hline & \multicolumn{2}{c}{ Disease severity $(\%)^{\mathbf{a}}$} \\
\cline { 2 - 3 } Treatment & Trial 1 & Trial 2 \\
\hline Open box & & \\
Control & $56.3 \pm 6.3$ & $9.3 \pm 5.2$ \\
No fungicide & $0.0 \pm 0.0$ & $0.7 \pm 0.7$ \\
Basal fungicide & $3.3 \pm 3.3$ & $1.3 \pm 0.7$ \\
Distal fungicide & $4.1 \pm 3.9$ & $0.1 \pm 0.1$ \\
Closed box & \\
Control & $66.1 \pm 11.4$ & $6.2 \pm 3.4$ \\
No fungicide & $0.0 \pm 0.0$ & $1.0 \pm 1.0$ \\
Basal fungicide & $0.0 \pm 0.0$ & $0.0 \pm 0.0$ \\
Distal fungicide & $0.0 \pm 0.0$ & $0.0 \pm 0.0$ \\
\hline a Percentage of lower leaf surface supporting \\
sporulation, \pm one standard error of the mean. \\
b Five leaves per seedling were inoculated with \\
Plasmopara viticola, and some were treated \\
with mefenoxam on the two most distal or \\
basal of these leaves 48 h later. One seedling \\
from each fungicide treatment was placed with \\
the others in a box open to ambient air move- \\
ment or enclosed in clear plastic bags. As \\
controls, additional inoculated seedlings were \\
placed in another room to avoid exposure to \\
mefenoxam vapor.
\end{tabular}


ment of guidelines for timing and placement of fungicide applications. In the absence of resistance, mefenoxam generally is regarded as one of the most effective compounds ever developed for control of grapevine downy mildew, and resistance management practices should be used to protect this material. In addition to its high intrinsic activity, its ability to potentially reach a grape cluster deep inside a canopy through translocation or vapor activity may, in part, explain its high efficacy under commercial vineyard conditions. To take full advantage of the acropetal systemic movement, lower, interior parts of the vine should be covered adequately, because merely covering leaves will not lead to systemic protection of clusters or other leaves. However, as indicated in our experiments, vapor activity also can compensate for suboptimal spray coverage, and should not be tissue dependent.

\section{LITERATURE CITED}

1. Cohen, Y., and Coffey, M. D. 1986. Systemic fungicides and the control of oomycetes. Annu. Rev. Phytopathol. 24:311-338.

2. Cohen, Y., Reuveni, M., and Eyal, H. 1979. The systemic antifungal activity of Ridomil against Phytophthora infestans on tomato plants. Phytopathology 69:645-649.

3. Ioannou, N., and Grogan. 1984. Control of Phytophthora root rot of processing tomato with ethazol and metalaxyl. Plant Dis. 68:429435.

4. Kennelly, M. M, Gadoury, D. M, Wilcox W. F., Magarey, P. A., and Seem, R.C. 2005. Seasonal development of ontogenic resistance to downy mildew in grape berries and rachises. Phytopathology 95:1445-1452.

5. Kennelly, M. M., Gadoury, D. M., Wilcox, W. F., Magarey, P. A., and Seem, R. C. 2007. Primary infection, lesion productivity, and survival of sporangia in the grapevine downy mildew pathogen, Plasmopara viticola. Phytopathology 97:512-522.

6. Kerkenaar, A., and Sijpesteijn, A. K. 1981. Antifungal activity of metalaxyl and furalaxyl. Pestic. Biochem. Physiol. 15:71-78.
7. Lafon, R., and Clerjeau, M. 1988. Downy mildew. Pages 11-13 in: Compendium of Grape Diseases. A. C. Goheen, ed. The American Phytopathological Society, St. Paul, MN.

8. Rowe, R. C. 1982. Translocation of metalaxyl and RE26745 in potato and comparison of foliar and soil application for control of Phytophthora infestans. Plant Dis. 66:989-993.

9. Staub, T., Dahmen, H., and Schwinn, F. J. 1978. Biological characterization of uptake and translocation of fungicidal acylalanines in grape and tomato plants. J. Plant Dis. Prot. 85:162-168.

10. Szkolnik, M. 1981. Physical modes of action of sterol-inhibiting fungicides against apple diseases. Plant Dis. 65:981-985.

11. Wong, F. P., and Wilcox, W. F. 2001. Comparative physical modes of action of azoxystrobin, mancozeb, and metalaxyl against Plasmopara viticola (grapevine downy mildew). Plant Dis. 85:649-656.

12. Zaki, A. I., Zentmyer, G. A., and LeBaron, H. M. 1981. Systemic movement of ${ }^{14} \mathrm{C}$-labeled metalaxyl in tomato, avocado, and Persea indica. Phytopathology 71:509-514. 